\title{
Perturbação de Hiperatividade e Défice de Atenção em Idade Pediátrica: Papel dos Cuidados de Saúde Primários
}

\section{Attention Deficit Hyperactivity Disorder in Pediatric Ages: Role of Primary Health Care}

Maria Inês Marques ${ }^{1}$, Joana Matias², Raquel Machado ${ }^{3}$, Ana Duarte², Maria José Fonseca², José Paulo Monteiro²

Autor Correspondente:

Maria Inês Marques [mariainesmarques@gmail.pt] Rua Actor João Rosa n4, $2^{\circ}$ direito, 1900-021 Lisboa, Portugal

\section{RESUMO}

A perturbação de hiperatividade/défice de atenção (PHDA) é uma das perturbações do desenvolvimento mais frequentes em idade pediátrica. Caracteriza-se por manifestações comportamentais que afetam o funcionamento e de-senvolvimento da criança/adolescente, em diferentes contextos. Os médicos de família são essenciais na abordagem da PHDA, pela aliança que estabelecem com a família e pelo seguimento regular em consulta de saúde infantil. Neste artigo descrevemos as principais características da PHDA, valorizando aspetos relacionados com as comorbilidades, diagnóstico e intervenção terapêutica, mas sobretudo com a atuação do médico de família na identificação precoce desta perturbação, na sua sinalização aos serviços especializados e no seguimento destes doentes.

PALAVRAS CHAVE: Criança; Cuidados de Saúde Primários; Transtorno de Déficit de Atenção com Hiperatividade

\section{ABSTRACT}

Attention deficit hyperactivity disorder (ADHD) is one of the most frequent developmental disorders in pediatric ages. It is characterized by behavioral manifestations that affect the functioning and development of the child/ adolescent, in different contexts. Family doctors are essential in approaching ADHD, by establishing a partnership with the family and due to regu-lar follow-up on child health consultation. In this article, the authors describe the main characteristics of $A D H D$, including comorbidities, diagnosis and therapeutic intervention, but above all the role of family doctors in screening this disorder and referencing to specialized services but also in the follow-up of these patients.

KEYWORDS: Attention Deficit Disorder with Hyperactivity; Child; Primary Health Care 


\section{INTRODUÇÃO}

A perturbação de hiperatividade e défice de atenção (PHDA), segundo a classificação internacional Diagnostic and Statistical Manual of Mental Disorders (DSM-5) da Academia Americana de Psiquiatria, ou perturbação hipercinética, segundo a International Classification of Diseases (ICD-10) da Organização Mundial de Saúde, é uma das perturbações do neurodesenvolvimento mais frequentes em idade pediátrica, persistindo em muitos casos na idade adulta. É uma patologia complexa, heterogénea, multifatorial, com grande impacto no indivíduo, na família e na sociedade.1-3

O diagnóstico e tratamento precoces da PHDA são determinantes para o seu prognóstico. Reduzem a intensidade dos sintomas, melhoram o rendimento académico e laboral, facilitam a integração social e diminuem a ocorrência de outras perturbações, que frequentemente se associam. Os médicos de Medicina Geral e Familiar (MGF), pelo estreito conhecimento que têm com a criança e a sua família nos cuidados primários de saúde, têm um papel fulcral na abordagem desta patologia. ${ }^{4}$

Para além de uma breve revisão sobre esta perturbação, os autores pretendem abordar a atuação do médico de família na sua identificação precoce, na sinalização aos serviços especializados e no seguimento destes doentes.

\section{DEFINIÇÃO}

A PHDA foi descrita pela primeira vez como entidade clínica distinta em 1968 na segunda edição do DSM, sob a designação de distúrbio hipercinético da infância. Desde então, a investigação científica tem conduzido a atualizações sucessivas da sua definição. ${ }^{4,5}$

Genericamente, a PHDA define-se como uma perturbação do neurodesenvolvimento caracterizada pela presença de três grupos de sintomas: défice de atenção (dificuldade em dirigir e manter a atenção e pouca persistência para a realização das tarefas), hiperatividade (agitação motora excessiva e desadequada) e impulsividade (dificuldade em gerir os impulsos e em protelar a recompensa). Estes sintomas terão de persistir pelo menos durante seis meses, em dois ou mais contextos (casa e escola, por exemplo), ser inconsistentes com a idade e o nível de desenvolvimento do indivíduo e interferir significativamente com o seu funcionamento. ${ }^{1-3,5-8}$

Secundariamente, podem associar-se outros sintomas com importante impacto prognóstico: ineficácia na realização das tarefas, insucesso académico ou profissional, baixa tolerância à frustração, baixa autoestima, labilida- de emocional, isolamento social e exposição a situações de risco. ${ }^{3-5,9}$

O quadro clínico é semelhante na idade escolar, adolescência e idade adulta, verificando-se, no entanto, um decréscimo progressivo da intensidade da hiperatividade, mantendo-se a impulsividade e, sobretudo, o défice de atenção. $7,8,10$

\section{EPIDEMIOLOGIA}

As estimativas de prevalência da PHDA publicadas internacionalmente são muito heterogéneas, dependendo da amostra estudada, da metodologia aplicada e dos critérios de diagnóstico utilizados. ${ }^{4}$

O DSM-IV-TR estimava que 3\% a 7\% das crianças em idade escolar teriam o diagnóstico de PHDA; o DSM-5 estima que $5 \%$ das crianças e 2,5\% dos adultos apresentem este diagnóstico; a ICD-10, utilizando critérios mais restritos, estima uma prevalência de 1\% a 2\% entre os 5 e os 16 anos de idade.?

Em Portugal não existem dados precisos, estando publicados alguns trabalhos que sugerem taxas de prevalência de aproximadamente $4 \%$ a 5\%, à semelhança do descrito por outros países. ${ }^{4}$

Globalmente, a prevalência parece ter-se mantido estável nos últimos anos, tendo aumentado apenas a frequência do diagnóstico. Ainda assim, estima-se que muitos casos não estejam adequadamente identificados. ${ }^{4,7}$

É uma patologia mais frequentemente diagnosticada na idade escolar e adolescência, em função da maior exigência social e académica. ${ }^{2,3}$ No entanto, pode-se manifestar em idades mais precoces, embora antes dos quatro anos os sintomas de PHDA sejam difíceis de distinguir dos comportamentos habituais dessas idades.

É mais prevalente no sexo masculino, embora na adolescência e idade adulta este predomínio seja menos notório. Alguns autores contestam esta afirmação, defendendo que no sexo feminino pode existir um subdiagnóstico, por predominarem os sintomas de desatenção, com uma expressão clínica mais tardia e menos exuberante. $2,3,10$

\section{FISIOPATOLOGIAE ETIOLOGIA}

Na base fisiopatológica da PHDA, vários estudos destacam a existência de alterações da anatomia (menor volume cerebral) e fisiologia do sistema nervoso central, particularmente do córtex pré-frontal, gânglios da base e cerebelo, com menor disponibilidade de neurotransmissores na fenda sináptica (dopamina e noradrenalina). 5,7,8 
Quanto à etiologia exata da PHDA, apesar dos inúmeros trabalhos realizados, esta permanece por esclarecer. No entanto, admite-se ser uma patologia de natureza multifatorial, resultante da interação de fatores de risco indi-



\section{FATORES DE RISCO INDIVIDUAIS:}

A. fatores genéticos, em 25\% a 90\% dos casos existe história familiar de PHDA, sobretudo irmãos e/ou pai; têm sido implicados vários genes, nomeadamente dos recetores e transportadores dos neurotransmissores $^{10,11}$;

B. outras doenças neurológicas, como epilepsia, sobretudo se associada a perturbação do desenvolvimento intelectual, perturbações do espetro do autismo ou síndromes neurocutâneas;

c. fatores associados ao período pré e perinatal, como a exposição a tabaco, álcool e drogas na gravidez, prematuridade, baixo peso ao nascer;

D. fatores pós-natais: traumatismo cranioencefálico grave, infeção do sistema nervoso central, exposição a agentes tóxicos como organofosfatos e metais pesados.

\section{FATORES DE RISCO FAMILIARES:}

A. tipo de vinculação; insegura e desorganizada;

B. disfunção familiar: privação afetiva grave e precoce, conflitualidade intrafamiliar, violência doméstica, estilo parental permissivo;

c. patologia psiquiátrica parental, como a perturbação do humor materna, comportamentos aditivos.

\section{FATORES DE RISCO AMBIENTAIS:}

A. família numerosa;

B. baixo nível socioeconómico e cultural;

C. acolhimento institucional.

De notar, que os fatores de risco nomeados não são específicos da PHDA, associando-se também à expressão de outras perturbações neuropsiquiátricas. ${ }^{7}$

\section{CRITÉRIOS DE DIAGNÓSTICO}

Apesar da reconhecida natureza neurobiológica da PHDA, atualmente ainda não existe nenhum marcador biológico disponível. Assim, o diagnóstico é exclusivamente clínico, baseando-se numa cuidadosa história clínica e observação. ${ }^{1,5,7,8}$

Para ajudar a um diagnóstico mais preciso, é possível a utilização dos critérios publicados pela Academia Americana de Psiquiatria no DSM-5 e pela Organização Mundial de Saúde na ICD-10. Os critérios são idênticos nos dois sistemas classificativos, embora a ICD-10 exija a presença simultânea de sintomas de desatenção e hiperatividade para o diagnóstico; por outro lado, o DSM fundamenta-se numa revisão mais extensa da literatura. ${ }^{1,2,5}$ Assim, são mais frequentemente recomendados a utilização dos critérios publicados no manual da Academia Americana de Psiquiatria.

Os critérios de diagnóstico da PHDA segundo a quinta e mais recente versão do DSM (publicada em 2013) são apresentados na Tabela 1.6 Comparando com a versão anterior, no DSM-5 a idade de início dos sintomas foi alargada dos 7 para os 12 anos. Adicionalmente, o número de sintomas necessário para o diagnóstico nos adolescentes (com idade igual ou superior a 17 anos) e adultos foi reduzido de seis para cinco, facilitando o diagnóstico nesta população. Paralelamente, o DSM-5 passou a permitir o diagnóstico simultâneo de PHDA e perturbação do espectro do autismo. Os três subtipos de PHDA mantiveram-se, passando a designar-se por "apresentações", que correspondem aos sintomas predominantes na altura do diagnóstico e a contemplar três especificadores de gravidade, reconhecendo a diversidade e variação temporal da sintomatologia da PHDA. Nos restantes aspetos os critérios de PHDA no DSM-5 são sobreponíveis ao do DSM-IV-TR. 4,6,7

De referir, que para o correto diagnóstico de PHDA, este deve ser baseado no relato de diferentes observadores, pois os sintomas devem estar presentes em pelo menos dois contextos diferentes. Outro aspeto a caracterizar é a qualidade do funcionamento social, académico ou ocupacional, uma vez que para o diagnóstico de PHDA pressupõe-se a interferência dos sintomas nestes domínios. Importa também destacar que os sintomas não podem ocorrer devido a outra patologia do desenvolvimento, esquizofrenia, psicose ou doença mental. ${ }^{6}$

\section{AVALIAÇÃO NA CONSULTA DE SAÚDE INFANTIL}

Os médicos de MGF são essenciais na prevenção e identificação precoces de patologias neuropsiquiátricas, na colaboração com as outras especialidades e na criação de alianças terapêuticas com as famílias. ${ }^{9,12}$ Se as equipas hospitalares têm competências específicas na área da saúde mental infanto-juvenil, são os Cuidados de Saúde Primários que estão vocacionados para uma intervenção na comunidade, tanto pelo conhecimento das famílias e sua trajetória de vida, como pelo conhecimento das necessidades e recursos locais. ${ }^{12,13}$

Assim, dada a elevada prevalência da PHDA, a todas as crianças e adolescentes com sintomas de défice de 
atenção, hiperatividade, impulsividade, mau rendimento escolar e/ou problemas de comportamento deve ser realizada uma avaliação cuidadosa, incluindo os critérios de PHDA para o diagnóstico ou exclusão desta patologia. 1,9,10,14 $\mathrm{O}$ médico de MGF deverá estar bem familiarizado com este diagnóstico, sendo habitualmente a eles que os pais recorrem inicialmente. 1,4, $^{1,9}$

Segundo as recomendações do Reino Unido do National Institute for Health and Care Excellence (NICE), seguidas por vários países europeus, os médicos de MGF têm a responsabilidade de identificar os indivíduos com suspeita de PHDA e de encaminhá-los para os serviços secundários especializados. No entanto, não Ihes é pedido que confirmem o diagnóstico, nem iniciem o tratamento. 12,15 Já a Associação Americana de Pediatria afirma que a avaliação diagnóstica formal da PHDA (após os quatro anos de idade) e a implementação de um plano terapêutico poderá ser realizada pelo médico de MGF. Na defesa desta posição, assume que delegar todas as perturbações neuropsiquiátricas para especialistas de saúde mental não é viável, devendo a referenciação ser feita apenas pontualmente. ${ }^{16}$

Os autores do presente artigo identificam-se com as recomendações publicadas pelo NICE. Acreditamos

TABELA 1. Critérios de Diagnóstico da PHDA segundo o DSM-5. ${ }^{6}$

CRITÉRIO A. Seis (ou mais) dos seguintes sintomas de desatenção e/ou hiperatividade-impulsividade (duração mínima de seis meses), de forma claramente inconsistente com o nível de desenvolvimento e que afeta diretamente as atividades sociais e académicas/laborais. Para indivíduos com idade igual ou superior a 17 anos são necessários pelo menos cinco dos seguintes sintomas.

\section{DESATENÇÃO:}

A. Frequentemente, falha em dar atenção suficiente aos pormenores ou comete erros por falta de atenção na escola, no trabalho ou em outras atividades.

B. Frequentemente, tem dificuldade em manter a atenção no desempenho de tarefas ou atividades.

C. Frequentemente, parece não ouvir quando se lhe fala diretamente, mesmo na ausência de uma distração óbvia.

D. Frequentemente, não segue as instruções e não termina os trabalhos escolares, as tarefas domésticas ou as tarefas profissionais.

E. Frequentemente, tem dificuldade em organizar tarefas e atividades.

F. Frequentemente, evita, não gosta ou está relutante em iniciar tarefas que requeiram atenção.

G. Frequentemente, perde objetos necessários para as tarefas ou atividades diárias ou esquece-se de compromissos.

H. Frequentemente, é facilmente distraído com estímulos irrelevantes.

I. Frequentemente, esquece-se das atividades quotidianas.

\section{HIPERATIVIDADE E IMPULSIVIDADE:}

A. Frequentemente, agita ou bate com as mãos e os pés ou remexe-se quando está sentado.

B. Frequentemente, levanta-se em situações em que se espera que esteja sentado.

C. Frequentemente, corre ou salta de forma excessiva em situações em que é inadequado fazê-lo (nos adolescentes mais velhos e adultos, tal pode corresponder a sentir-se inquieto).

D. Frequentemente, é incapaz de jogar ou envolver-se com tranquilidade em atividades de lazer.

E. Está frequentemente «em movimento», agindo como se estivesse «ligado a um motor».

F. Frequentemente, fala em excesso.

G. Frequentemente, precipita as respostas antes da pergunta ser completada.

H. Frequentemente, tem dificuldade em esperar pela sua vez.

I. Frequentemente, interrompe ou interfere nas atividades dos outros.

CRITÉRIO B. Vários dos sintomas presentes antes dos 12 anos de idade.

CRITÉRIO C. Vários dos sintomas de desatenção ou hiperatividade/impulsividade estão presentes em dois ou mais contextos (por exemplo, em casa e na escola ou trabalho; com familiares e amigos).

CRITÉRIO D. Os sintomas interferem com a qualidade do funcionamento social, académico ou ocupacional.

CRITÉRIO E. Os sintomas não ocorrem exclusivamente durante o curso de esquizofrenia ou outra perturbação psicótica e não são mais bem explicados por outra perturbação mental (por exemplo, perturbação do humor, perturbação da ansiedade, perturbação dissociativa, perturbação da personalidade ou intoxicação/abstinência de substâncias de abuso).

\section{Tipo de apresentação:}

- APRESENTAÇ̃̃O COMBINADA: nos últimos seis meses, estão preenchidos o CRITÉRIO A 1) (Desatenção) e o CRITÉRIO A 2) (Hiperatividade e Impulsividade).

- APRESENTAÇÃO PREDOMINANTEMENTE COM DESATENÇÃO: nos últimos seis meses, está preenchido o CRITÉRIO A 1) (Desatenção), mas não o CRITÉRIO A 2) (Hiperatividade e Impulsividade).

- APRESENTAÇÃO PREDOMINANTEMENTE COM HIPERATIVIDADE E IMPULSIVIDADE: nos últimos seis meses, está preenchido o CRITÉ RIO A 2) (Hiperatividade e Impulsividade), mas não o CRITÉRIO A 1 (Desatenção). 
que o papel do médico de MGF deverá ser de rastreio, referenciação dos casos suspeitos, e depois um seguimento em estreita colaboração no apoio no tratamento e monitorização clínica.9,12,13,15 Esta referenciação deverá ser imediata na presença de sintomas moderados ou graves. ${ }^{15}$ Perante situações com menor impacto funcional, o médico de MGF poderá oferecer aos cuidadores estratégias de gestão comportamental, incluindo acompanhamento em consulta de Psicologia ao nível da comunidade (seja no Centro de Saúde, Junta de Freguesia ou Instituições locais) e reavaliando posteriormente ao fim de um período máximo de 10 semanas. Caso os sintomas persistam, a referenciação deverá ser realizada. $9,13,15,17$

O diagnóstico formal de PHDA exige a abordagem por uma equipa multidisciplinar experiente, incluindo um pediatra do desenvolvimento e/ou neuropediatra, por vezes um psicólogo (nomeadamente quando se suspeita de perturbações específicas da aprendizagem ou défice cognitivo associados) e um pedopsiquiatra (sobretudo perante alterações graves do comportamento, comportamentos bizarros e agressividade excessiva). 2,4,13,15

A avaliação diagnóstica inclui: 1) entrevista clínica: antecedentes familiares e pessoais, caracterização dos sintomas, do percurso escolar, do funcionamento social e do ambiente familiar; 2) exame objetivo, incluindo exame neurológico e avaliação do desenvolvimento psicomotor; 3) avaliação da visão e audição; 4) observação comportamental direta (embora o comportamento em consulta possa não ser exemplificativo do que ocorre nos contextos mais habituais); 5) avaliação psicológica, quando necessário; 6) aplicação de escalas de avaliação comportamental a pais, professores (escala de Conners, por exemplo) e, em alguns casos, à própria criança e jovem. ${ }^{1,3-5,7,8,10,14}$

O uso de exames complementares de diagnóstico (laboratoriais ou imagiológicos) não deve ser feito por rotina, sendo apenas útil para a exclusão de patologias médicas subjacentes (nomeadamente hipertiroidismo, epilepsia e alteração da acuidade visual ou auditiva) e apenas se a história e o exame objetivo o sugerir., ${ }^{3,7} \mathrm{O}$ doseamento de neurotransmissores ou de outro tipo de análise não tem qualquer evidência científica na avaliação da PHDA.7,18,19

Enquanto aguarda esta avaliação formal, o médico de MGF poderá implementar medidas gerais e estratégias comportamentais. ${ }^{9,14}$ Após a avaliação pela equipa mais diferenciada, poderá ajudar na vigilância dos sintomas de PHDA, das comorbilidades e dos efeitos do tratamento e no apoio à prescrição.

As crianças e adolescentes com PHDA beneficiam sig- nificativamente de uma gestão integrada e multidisciplinar desta perturbação. Nos indivíduos em que a família, escola, médico de MGF e médicos especializados trabaIham em conjunto o prognóstico a longo prazo é muito melhor. ${ }^{9}$

\section{ESCALAS DE CONNERS}

As escalas de avaliação comportamental, ao permitirem caracterizar o comportamento do indivíduo nos seus diferentes contextos, acrescentam preciosa informação à avaliação realizada pelos profissionais de saúde, ajudando no diagnóstico de PHDA. No entanto, devem ser sempre assumidas como uma ferramenta auxiliar e não como um instrumento de diagnóstico per si.3,7

As escalas de Conners, muito estudadas e divulgadas, surgiram em 1969 com o objetivo de avaliar a resposta clínica das crianças com PHDA ao tratamento farmacológico. Atualmente, assumem um papel mais alargado, sendo as escalas mais frequentemente utilizadas, para avaliação da presença e gravidade dos sintomas de PHDA. ${ }^{3,5}$

As versões reduzidas das escalas de Conners para pais e professores, validadas para a população portuguesa e de mais fácil aplicação que as versões completas, são bons instrumentos para o rastreio de PHDA, ,3 cujos resultados deverão conduzir a uma rigorosa avaliação multidisciplinar, de forma a confirmar ou excluir a suspeita diagnóstica. ${ }^{5}$

\section{COMORBILIDADES}

A avaliação na consulta de saúde infantil deverá pretender não apenas diagnosticar PHDA, mas também excluir diagnósticos alternativos e identificar comorbilidades. A inespecificidade dos sintomas de PHDA e a sua sobreposição com o de outras patologias dificulta o diagnóstico diferencial. .,7 $^{2}$ S diagnósticos que podem mimetizar ou coexistir com a PHDA são apresentados na Tabela 2.2,6,14

Cerca de um terço a 60\% das crianças com PHDA apresentam outras comorbilidades. ${ }^{2,20}$ A sua presença agrava o quadro clínico, dificulta o diagnóstico e a abordagem terapêutica da PHDA, devendo ser ativamente pesquisada. $2,3,4,7$

Algumas perturbações do neurodesenvolvimento (da linguagem e da aprendizagem, por exemplo), a perturbação de ansiedade, os tiques e a perturbação de oposição/desafio emergem no início do desenvolvimento infantil. Em contraste, as perturbações do humor (nomeadamente depressão) e as perturbações graves do comportamento surgem no final do ensino básico e na transição para a adolescência. A partir desta idade, a 
PHDA associa-se a um risco acrescido para consumo de substâncias psicoativas e à emergência de perturbações da personalidade. 1,4,7, $, 14,21^{-1}$

\section{TRATAMENTO}

No tratamento da PHDA, a intervenção multimodal combinada é comprovadamente superior às intervenções terapêuticas isoladas. Deverá ser realizada uma abordagem funcional abrangente que envolva a família, a escola e a comunidade, estabelecendo uma estratégia transversal no tempo e nos espaços, paralelamente à abordagem sintomática, com tratamento farmacológico, quando necessário. ${ }^{22,23}$

\section{MEDIDAS GERAIS}

médico assistente da criança ou adolescente com PHDA tem um papel fundamental na educação dos cuidadores relativamente às características desta patologia, no ensino de estratégias comportamentais e no estabelecimento de expectativas realistas. Deverá promover a criação de rotinas bem definidas, incentivar o estabelecimento de regras claras e de recompensas ou castigos consistentes e imediatos, a valorização dos desempenhos positivos e promover uma relação próxima e afetuosa entre a criança e os pais.

Relativamente à escola, poderá aconselhar os cuidadores na articulação com os professores, nomeadamente com a figura de referência, responsável por ajudar a conter e organizar o comportamento da criança em contexto escolar. Deverá incentivar a promoção de estratégias pedagógicas de intervenção e de avaliação adaptadas às necessidades individuais, por exemplo, a integração no horário da manhã e numa turma com número reduzido de alunos; estando ciente das circunstâncias de elegibilidade para Plano Educativo Individual (PEI) ao abrigo do decreto 3/2008 artigo 18:3,13,14,16 Necessita igualmente de identificar a existência de dificuldades associadas que exijam uma intervenção terapêutica específica, como terapia da fala ou psicomotricidade.

Tanto nos contextos familiar e académico, deverá promover o uso de ferramentas como a elaboração de um cronograma com as rotinas diárias, exposto num local visível, ou a utilização de organizadores do material escolar e organizadores dos itens do dia a dia. ${ }^{24}$

No grupo dos adolescentes, poderá oferecer orientações específicas relativamente ao percurso académico e às relações familiares e sociais. Deverá alertar para o maior risco de isolamento social e de comportamentos de risco (acidentes de viação, abuso de substâncias), tentando preveni-los. 5,15
Não esquecer os outros contextos em que a criança ou adolescente se move, como as atividades extra-escolares, família alargada, etc.

Algumas fontes adicionais de informação podem ser obtidas na internet, como no site do Clube PHDA (disponível em www.clubephda.pt).

\section{TERAPÊUTICA FARMACOLÓGICA}

O metilfenidato (MFD) é o fármaco mais utilizado no tratamento da PHDA, tendo uma eficácia descrita de aproximadamente 80\% na redução dos sintomas nucleares. É um fármaco estimulante, cujo mecanismo de ação consiste no bloqueio da recaptação de dopamina e noradrenalina na fenda sináptica. ${ }^{23}$

O MFD está disponível sob três formulações: uma de libertação imediata (LI) e duas de libertação prolongada (LP); as características farmacológicas destas três formulações são apresentadas na Tabela 3.9,14

Este fármaco está aprovado para utilização a partir dos seis anos de idade, apresentando uma elevada segurança e tolerabilidade. No entanto, a sua administração poderá ser considerada em crianças mais novas (a partir dos quatro anos), em casos pontuais e orientados pelo especialista. ${ }^{16}$

Os efeitos adversos mais frequentes do MFD são as perturbações do sono (insónia), as cefaleias, a anorexia e a dor abdominal. Os tiques, descrevendo-se um agravamento de tiques pré-existentes em 20\% dos doentes;

TABELA 2. Patologias que podem mimetizar ou coexistir com a PHDA. $2,6,14$

Diminuição da acuidade auditiva
Diminuição da acuidade visual
Epilepsia
Hipertiroidismo
Doenças genéticas (síndrome do X frágil, neurofibromatose,
síndrome de deleção 22q11)
Síndrome fetal alcoólico
Síndrome de Gilles De La Tourette
Perturbação específica da linguagem
Perturbação específica da aprendizagem (leitura,
expressão escrita ou matemática)
Perturbação do espectro do autismo
Perturbação do desenvolvimento intelectual
Perturbação de oposição/desafio
Perturbação do comportamento
Perturbação de ansiedade generalizada/de separação
Perturbação bipolar
Perturbação depressiva persistente/depressiva major
Perturbação obsessivo-compulsiva
Perturbação do sono (apneia obstrutiva do sono, síndrome das
pernas inquietas, distúrbio periódico do movimento dos membros)


TABELA 3. Características farmacológicas das três formulações do MFD. , $14^{-14}$

\begin{tabular}{|c|c|c|c|}
\hline & $\begin{array}{l}\text { Libertação Imediata } \\
\quad\left(\text { Ex: Rubifen }{ }^{\circledR}\right)\end{array}$ & $\begin{array}{l}\text { Libertação Prolongada } \\
\left.\text { (Ex: Ritalina }{ }^{\circledR}\right)^{*}\end{array}$ & $\begin{array}{l}\text { Libertação Prolongada } \\
\qquad\left(\text { Ex: Concerta }{ }^{\circledR}\right)^{+}\end{array}$ \\
\hline Início de ação & 30 minutos & 60 minutos & 30 minutos \\
\hline Duração média & 3 a 4 horas & 6 a 8 horas & 8 a 12 horas \\
\hline Apresentação & 5,10 e $20 \mathrm{mg}$ & 20,30 e $40 \mathrm{mg}$ & $18,27,36$ e $54 \mathrm{mg}$ \\
\hline Dose máxima & $60 \mathrm{mg} / \mathrm{dia}$ & $60 \mathrm{mg} / \mathrm{dia}$ & $54 \mathrm{mg} / \mathrm{dia}$ \\
\hline Vantagens & $\begin{array}{c}\text { Preço; menos efeitos adversos } \\
\text { (sono, apetite) }\end{array}$ & $\begin{array}{l}\text { Pode-se abrir a cápsula } \\
\text { e misturar com comida mole }\end{array}$ & Perfil mais "fisiológico" \\
\hline Desvantagens & $\begin{array}{c}\text { Mais de } 1 \text { toma diária } \\
\text { (estigmatização e menor adesão) }\end{array}$ & & Não pode ser fragmentado; preço \\
\hline
\end{tabular}

* Mecanismo SODAS (Spheroidal Oral Drug Absorption System): 50\% de libertação imediata e 50\% libertação retardada - tempo bimodal com 2 picos distintos separados por cerca de $4 \mathrm{~h}$.

+ Mecanismo OROS (Osmotic Release Control System).

as alucinações e outras perturbações psicóticas e as alterações do humor, mais frequentes nas crianças mais novas, são efeitos adversos muito menos frequentes. Podem igualmente ocorrer discretas elevações da frequência cardíaca e pressão arterial, raramente significativas. Por outro lado, é possível verificar-se uma ligeira diminuição da velocidade de crescimento, com uma altura final prejudicada em um a dois centímetros, principalmente na utilização prolongada de doses elevadas. 16,24,25 A maioria dos efeitos adversos são dose-dependentes e temporários, pelo que raramente obrigam à suspensão do fármaco. São controláveis através de uma monitorização frequente, alteração da dose e, se necessário, da formulação.

História de hipertiroidismo, doença cardiovascular e/ ou doença psiquiátrica grave contraindicam, de forma absoluta ou relativa, o uso de MFD. Não há evidência clara de aumento do risco de morte súbita associada ao MFD. ${ }^{16,23,26}$ Nos casos com história pessoal de doença cardíaca, palpitações ou síncope, história familiar de morte súbita, síndrome de Wolff-Parkinson-White, cardiomiopatia hipertensiva ou síndrome do QT longo ou alterações no exame cardiovascular, deve-se realizar um eletrocardiograma e/ou referenciar à consulta de Cardiologia Pediátrica antes de se iniciar este fármaco. ${ }^{16}$

É administrado na dose de 0,2 a $2 \mathrm{mg} / \mathrm{kg} / \mathrm{dia}$, em toma única (se formulação de LP) ou até três tomas diárias (se formulação de LI). Regra geral, inicia-se com doses baixas da formulação de LI, o que permite observar a resposta ao tratamento, aumentando-se semanalmente até à dose mínima eficaz, ao longo de quatro a seis semanas. $^{25}$

Poderá ser usado de forma descontínua, apesar de teoricamente fazer mais sentido o uso de forma contínua pois a PHDA não tem só repercussões negativas na escola. Também o grupo de amigos, a família alargada e as atividades extraescolares têm um peso significativo na vida destas crianças ou adolescentes. Por isso, a decisão de medicar no fim-de-semana deve considerar o tipo de sintomas e o seu impacto nesses dias. Anualmente devem ser reavaliadas as indicações para a sua utilização, sugerindo-se a sua interrupção na segunda semana de janeiro; caso a suspensão resulte num significativo agravamento dos sintomas, o fármaco deverá ser reiniciado. ${ }^{25}$

A atomoxetina é um fármaco não estimulante, cujo mecanismo de ação consiste na inibição da recaptação de noradrenalina na fenda sináptica. É uma alternativa farmacológica nas crianças e adolescentes com má tolerância ao MFD, nomeadamente nos casos com tiques ou perturbação da ansiedade associados. Parece ter uma eficácia discretamente inferior ao MFD na redução dos sintomas nucleares da PHDA. Deve ser iniciada na dose de $0,5 \mathrm{mg} / \mathrm{kg} / \mathrm{dia}$, com aumento após sete dias para a dose de manutenção de $1,2 \mathrm{mg} / \mathrm{kg} / \mathrm{dia}$ (em uma ou duas tomas diárias). O efeito terapêutico é atingido após quatro a seis semanas de tratamento. Os efeitos adversos mais frequentes são sonolência e anorexia; alterações do humor e ideação suicida ocorrem muito raramente. A principal limitação ao seu uso é o seu elevado custo. ${ }^{16}$

Outros fármacos podem ser eficazes (por exemplo, os antidepressivos tricíclicos) como terapêutica de segunda linha. ${ }^{16}$

\section{SUPLEMENTOS}

Os ácidos gordos do grupo ómega 3 (ácido docosahexanoico), comercializados sob a forma de cápsulas, xarope ou espuma são considerados seguros pela Food and Drug Administration. No entanto, há pouca evidência científica da sua eficácia. Devem ser tomados diariamente e por um período mínimo de três meses. Não têm efeitos adversos e são uma alternativa para os pais que recusam o tratamento farmacológico.

Uma outra alternativa é a ingestão de um café por dia 
(no período da manhã), cujo efeito se estima durar duas horas.

É importante esclarecer de que não há comprovado benefício em restrições alimentares ou dietas específicas.

\section{TERAPIA COMPORTAMENTAL}

A terapia comportamental (intervenções que pretendem alterar o ambiente de forma a regular o comportamento) deve ser considerada de forma a melhorar-se o desempenho académico, as competências sociais e a autoestima das crianças e adolescentes. 2,9,14 Esta, em combinação com o MFD, demonstra efeitos muito positivos. A terapia comportamental assente no treino parental é, idealmente, o tratamento de primeira linha em crianças em idade pré-escolar. ${ }^{16}$ Lamentavelmente, em Portugal existem poucos programas de terapia comportamental validados, não sendo estes comparticipados pelo estado ou pelas seguradoras.

Poderá também haver lugar à terapia familiar, participação em grupos de pais e em grupos de apoio. Deverão ser sugeridas fontes de informação fidedignas e oferecidos folhetos informativos. ${ }^{25}$

\section{EVOLUÇÃO E PROGNÓSTICO}

O prognóstico da PHDA depende da gravidade dos sintomas no momento do diagnóstico e dos contextos familiar, escolar e social da criança ou adolescente. $\bigcirc$ diagnóstico e intervenção precoces são, sem dúvida, fatores determinantes para um melhor prognóstico a longo prazo. ${ }^{1,2}$

Segundo vários autores, em 10\% a 60\% dos casos os sintomas persistem na idade adulta. 1,9-11,27 $\mathrm{O}$ insucesso académico, associado à desadaptação social, causam sofrimento e baixa autoestima, contribuindo para um maior risco de disfunção familiar, laboral e social em adultos. ${ }^{28}$ Assim, a abordagem da PHDA em idade pediátrica tem um grande impacto em toda a sociedade.

\section{CONCLUSÃO}

A PHDA é a perturbação do neurodesenvolvimento mais frequente em idade pediátrica. Na maioria dos casos, é aos Cuidados de Saúde Primários que as crianças e adolescentes com esta patologia são inicialmente sinalizados pelos pais e/ou professores. $\bigcirc$ médico de MGF tem, por isso, um papel fundamental na identificação precoce desta perturbação e na sua sinalização aos serviços especializados.

A PHDA associa-se muito frequentemente a várias comorbilidades (nomeadamente alterações do compor- tamento) que podem mascarar a sua presença. Assim, perante a suspeita desta perturbação deve-se sempre realizar uma avaliação formal por uma equipa multidisciplinar experiente. Uma vez confirmado o diagnóstico, deve ser implementado um plano de tratamento integrado, incluindo educação da família, estratégias para gestão do comportamento e, eventualmente, tratamento farmacológico.

Os médicos de MGF são essenciais na abordagem da PHDA, desde a suspeição do diagnóstico à referenciação atempada, e finalmente na criação de uma aliança terapêutica com a família e professores e monitorização clínica.

CONFLITOS DE INTERESSE: Os autores declaram não ter qualquer conflito de interesse na realização do presente trabalho.

FONTES DE FINANCIAMENTO: Não houve qualquer fonte de financiamento na realização do presente trabalho.

CONFLICTS OF INTEREST: The authors declare that they have no conflicts of interest.

FINANCIAL SUPPORT: This work has not received any contribution, grant or scholarship.

\section{REFERÊNCIAS}

1. Fernandes E, António JP. Perturbação de hiperatividade com défice de atenção. Rev Port Clin Geral. 2004;20:451-4.

2. Pardilhão C, Marques M, Marques C. Perturbações do comportamento e perturbação de hiperatividade com défice de atenção: Diagnóstico e intervenção nos Cuidados de Saúde Primários. Rev Port Clin Geral. 2009;25:592-9.

3. Pereira dos Santos O. Avaliação da perturbação de hiperatividade e défice de atenção na adolescência [tese de dissertação]. Lisboa: Instituto Superior de Educação e Ciências; 2012.

4. Oliveira L, Pereira M, Medeiros M, Serrano A. PHDA: O que há de novo no DSM-5? Rev Port Pedag. 2015;49:75-94.

5. Carapeto O. Um novo olhar... à PHDA na sala de aula do $1^{\circ} \mathrm{Ci}-$ clo [dissertação]. Castelo Branco: Instituto Politécnico de Castelo Branco, Escola Superior de Educação; 2012.

6. American Psychiatric Association. Manual de diagnóstico e estatística das perturbações mentais. $5^{\text {a }}$ ed. Lisboa: Climepsi Editores; 2014.

7. Banaschewski T, Becker K, Döpfner M, Holtmann M, Rösler M, Romanos M. Attention-deficit/ hyperactivity disorder - A current overview. Dtsch Arztebl Int. 2017;114:149-59.

8. Afonso S. Perturbação da hiperactividade e défice de atenção. Cadernos Otorrinolaringologia. 2010;1-4. [acedido em 14/02/2018]. Disponível em: http://www.cadernosorl.com/ artigos/2/3.pdf

9. Kewley G, Halliwell N. Attention deficit hyperactivity disorder: Clinical update. Br J Gen Pract. 2014;243-5.

10. Filipe C. A perturbação de hiperatividade com défice de atenção no adulto. Rev Port Clin Geral. 2004;20:733-7.

11. Kates N. Attention deficit disorder in adults - Management in primary care. Can Fam Physician 2005; 51:53-9. 
12. Tatlow-Golden M, Prihodova L, Gavin B, Cullen W, McNicholas F. What do general practitioners know about ADHD? Attitudes and knowledge among first-contact gatekeepers: Systematic narrative review. BMC Fam Pract. 2016;17:1-15.

13. Coordenação Nacional para a Saúde Mental. Recomendações para a prática clínica da saúde mental infantil e juvenil nos cuidados de saúde primários. Lisboa: Alto Comissariado da Saúde, Ministério da Saúde [acedido em 10/06/2017]. Disponível em: http://www.dgs.pt/upload/membro.id/ficheiros/i015903.pdf

14. Felt BT, Bierdmann B, Christner JG, Kochhar P, Van Harrison R. Diagnosis and management of ADHD in children. Am Fam Physician. 2014;90:456-64.

15. National Institute for Health and Clinical Excellence 2008 (updated 2016). Attention deficit hyperactivity disorder: Diagnosis and management of ADHD in children, young people and adults (Clinical Guideline CG72) [acedido em 10/06/2017]. Disponível em: https://www.nice.org.uk/guidance/cg72

16. Subcommittee on Attention-Deficit/ Hyperactivity Disorder, Steering Committee on Quality Improvement and Management, Wolraich M, Brown L, Brown RT, DuPaul G, et al. ADHD: Clinical practice guideline for the diagnosis, evaluation, and treatment of attention-deficit/ hyperactivity disorder in children and adolescents. Pediatrics. 2011;128:1007-22.

17. National Institute of Mental Health 2008 (updated 2016). Attention Deficit Hyperactivity Disorder [acedido em 10/06/2017]. Disponível em: https://www.nimh.nih.gov/ health/topics/attention-deficit-hyperactivity-disorder-adhd/ index.shtml.

18. Ailts IA. Exploration of Neurotransmitter Levels and Attention-Deficit/Hyperactive Disorder [dissertação]. Menomonie: University of Wisconsin-Stout, The Graduate School; 2006.

19. Bergwerff CE, Luman M, Blom HJ, Oosterlaan J. No Tryptophan, Tyrosine and Phenylalanine Abnormalities in Children with Attention-Deficit/Hyperactivity Disorder. PLoS ONE. 2016;11:1-13.

20. Felt BT, Biermann B, Chrisner JG, Kochhar P. Harrison RV. Diagnosis and Management of ADHD in Children. Am Fam Physician. 2014;90:456-64.

21. Pliszka SR. Psychiatric comorbidities in children with attention deficit hyperactivity disorder: Implications for management. Paediatr Drugs. 2003;5:741-50.

22. Taylor E, Döpfner M, Sergeant J, Banaschewski T, Buitelaar J, CoghillD, et al. European clinical guidelines for hyperkinetic disorder - First upgrade. Eur Child Adolesc Psychiatry. 2004;13:17-30.

23. The MTA Cooperative Group. A 14-month randomized clinical trial of treatment strategies for attention-deficit/ hyperactivity disorder. Multimodal treatment study of children with ADHD. Arch Gen Psychiatry. 1999;56:1073-86.

24. Perrin JM, Friedman RA, Knilans TK, Black Box Working Group, Section on Cardiology and Cardiac Surgery. Cardiovascular monitoring and stimulant drugs for attention-deficit/ hyperactivity disorder. Pediatrics. 2008;122:451-3.

25. National Institute for Health and Care Excellence 2006 (updated 2009). Methylphenidate, atomoxetine and dexamfetamine for attention deficit hyperactivity disorder in children and adolescents (Technology appraisal guidance 98) [acedido em 10/06/2017]. Disponível em: https://www.nice.org.uk/guidance/ta98

26. Pliszka S, AACAP Work Group on Quality Issues. Practice parameter for the assessment and treatment of children and adolescents with attention-deficit/ hyperactivity disorder. J Am Acad Child Adolesc Psychiatry. 2007;46:894-921.
27. Faraone SV, Sergeant J, Gillberg C, Biederman J. The worldwide prevalence of ADHD: Is it an american condition? World Psychiatry. 2003;2:104-13.

28. DuPaul GJ, McGoey KE, Eckert TL, VanBrakle J. Preschool children with attention-deficit/ hyperactivity disorder: Impairments in behavioral, social, and school functioning. J Am Acad Child Adolesc Psychiatry. 2001;40:508-15. 